\title{
Comparison of Automated and Robotic Support Bodies for Building Facade Upgrading
}

\author{
K. Iturralde $1^{a}$, T. Linner $2^{\text {a }}$ T. Bock $3^{a}$ \\ ${ }^{a}$ Chair of Building Realization and Robotics, Technische_Universität_München, Germany \\ E-mail: kepa.iturralde@br2.ar.tum.de, thomas.linner@bri.arch.tu-muenchen.de, thomas.bock@br2.ar.tum.de
}

\begin{abstract}
The need for ameliorating the physical properties of existing buildings' envelopes is growing. For that purpose, a widely used solution is to add new layers on the external face. Each layer performs a function; insulation, moisture barrier or finishing. Energy harvesting devices can be installed on too. Each of these layers don't need to be assembled on site separately, there are already experiences with the installation of prefab components on the external face of existing façades. Those components can be accurately customized and adjusted to the geometrical irregularities of the existing building wall. Though, the installation of these prefab components is primarily based on manual tools and techniques. A robotic installing performance would facilitate the fixation of these new components on the exterior of the building. This means that the robotic tools must reach every point of the building's façade with enough stability and accuracy. Therefore, a proper support system (also known as robot body) is needed. Those supporting bodies should host different automated tools for installing the component. Before creating a structure or body from scratch, different existing support systems have been analyzed. First, there are the traditional support systems, where the operator works using manual tools. Second, robots and automated mechanisms that work on the exterior of the building have been studied. And third, technology in other fields has been taken into account. After the results of the virtual simulation have been gathered, those are compared with the data of traditional or manual installation methods. Thus, we can see if the solution is competitive enough in terms of cost and time. Besides, it is remarked the adequacy of each body type to work in different building typologies and it is also pointed out the suitability of each support body type for installing different façade components and materials. Finally, as a conclusion, the article defines possible future research.
\end{abstract}

Keywords -support body, façade, refurbishment

\section{Introduction}

The need for ameliorating the insulating properties of existing buildings is growing. For instance, the European Union is committed to accomplish an almost Zero-Energy consumption in the totality of the building stock by the year 2050 [1]. For this purpose, new insulating layers are added to the existing building, and sometimes, energy harvesting devices are installed on the building envelope. There are already experiences with the installation of prefab façade components on existing façades. Those components are accurately customized and adjusted to each of the irregularities in the geometry of the existing wall. But the installation of these prefab components is primarily based on manual tools and techniques.

We need to determine the Market Size in order to quantify the opportunities on the field. According to the BPIE organization, that assumes that there will be around 38 billion $\mathrm{m}^{2}$ useful floor area in 2050 [2], we can calculate that there will be 25 billion square meters of façade that need to be upgraded in Europe in order to fulfil with NEZB requirements. If we suppose, depending on the finishing and energy harvesting devices, the existing prize for upgrading these envelopes is around 100 euro per square meter of envelope, when installed manually, therefore, there is a potential market for 2,500 billion euro (in current prices) that needs to be invested on the building stock on the next decades till the year 2050, just to fulfil the NEZB objectives. In other words, it will mean the $0.6 \%$ of the total GDP, every year.

\section{Research methodology}

This study is part of a broader research about automated and robotic building envelope upgrading. This article focuses on the fixation of new façade components onto existing buildings. Those components are configured by multiple elements that fulfil different functions, such as insulation, moisture barrier, finishing and so on. This research assumes that the components are already designed and manufactured for being 
installed in a specific building geometry by robotic tools. In a parallel work of the research, the specifications of the component have been developed. Besides, the definition of the end-effectors is part of another phase of the research. Moreover, we must take into account that there might be some other solutions that are not based on fixing a component, but on printing or rendering a product. Those solutions are not taken into account in this paper, but must be analyzed in the future.

One issue to resolve is the component uploading. There must be a way to the place the component that has to be installed. As said before, in the case of building renovation, the material supply cannot be done from the interior, let's say, from the nearby window, but from the exterior, from the foot of the building. For an automated supply of components from the bottom of the building, it would be needed a hoist that could upload components. Another issue to solve is the accuracy of the positioning of the platform. An automated component fixation needs to be positioned with accuracy. Constant calibration and adjustment of the device is needed in order to perform accurately.

The main question of this specific research is if the existing devices can be readapted into robotic tools. Besides, we need to know which of the adapted versions fits best to a certain building typology.

The main goal of this specific research is the adaptation and development of the existing support structure into robot bodies that can host robotic tools.

How we can do that? The TRIZ [3] method can be used as a guideline. Even though not all the steps have been fulfilled, it has been a suitable tool. In order to approach this research, first, the existing supporting structures and devices have been analyzed. The analyzed support systems could be adapted for automatically adding a new component onto an existing façade, hence, a technological re-development is needed.

So second, the support body variants have been developed. Five main variants of support bodies have been conceived.

Three, the simulation of the performance of the developed variants has been carried out. The objective of this research is to compare the efficiency and competitiveness of these variants. In order to foresee the validity of the proposals, the performance of each has been tested in a qualitative way. To accomplish that, he used simulation software has been SolidEdge. Several parameters and facts have been checked. In one side, there are issues that are related to the operability of the robotic tool and each support body. Those are basically mechanical and kinematic topics. In the other side, the efficiency facts have been tested. Those are related to the competiveness of the automated installation process.

As a fourth step, the results of the simulation have been analyzed. Basically, we can see that the proposed systems fit better in certain situations.

And finally, a conclusion has been gathered and the direction of the future research has been defined. We can foresee that Tthe adopted solutions must be compared to traditional upgrading methods. But this will be thoroughly accomplished on the next phase of this research.

\section{Existing devices}

This sub-chapter paper will explain the existing auxiliary bodies and structures for reaching any part of the building. Every building has its specific requirements and therefore some systems are more appropriate than others in each case. Also, depending on the works that have to be done, one device may be more suitable than the rest. The support and upload systems are essential for executing the works on the external side of the building envelopes. Without them, it could be impossible to achieve any work. The support systems offer several advantages:

1. Accessibility in order to achieve the tasks to be fulfilled.

2. Material uploading for the maintenance or renewal procedures. Any works held in the façade of the building needs some material, and therefore, it needs to be uploaded to the place.

3. Risk prevention. Working on the heights must be a secure task. Therefore, some passive and active measures are taken.

4. Storage. The material arrives to the working place in big quantities and most of the times cannot be used directly of the façade, it must be stored for a while.

Not all the support systems have same characteristics; some of them are more suitable for some purposes than other. For instance, installation and desinstallation time of the support body is a fact that has to be considered before doing any job. There are minor tasks on the façade, like changing a drainage tube that are carried out in several hours. Installing a fixed scaffolding might not be suitable for this purpose. On the other side, before renting or acquiring a support system is necessary to calculate its investment costs and return according to the works that are going to be achieved. Adding a layer to the existing façade are intensive works and therefore movable cranes may result slow for this task. At least, under some circumstances, such as middle or high rises.

In this chapter, most common support systems will be briefly classified and, somehow, the possibility of the adaptation for achieving automated solutions will be foreseen: 


\subsection{Self supported bodies attached to the building}

In this section, we can find two main sub-variants. One is the Fixed scaffolding [4], which consists on attaching a fixed but provisional substructure to the existing building. In other words, an exterior structure is erected, close to the existing façade. It is probably the oldest method. They are also known as Supported Scaffolding Systems. Normally this substructure is made out of standard steel profiles. Most of the companies that produce standard scaffolding, they provide specific software for designing and budgeting [4]. This is very appropriate for the definition accuracy of the "scaffolding project", which is requested by the local authorities. Horizontal and vertical transportation is possible along this scaffolding. In façade renovation, it is quite common that material is lifted by elevators. Material Elevation systems can be adapted to almost any type of building [4]. Regarding to the components to be assembled on the façade, it has to be taken into account that they need to be smaller than the elevator and the modules of the scaffolding. Instead, the positioning and placing may result impossible.

On the other hand, the Mast climbing systems consists of a platform that is lifted up along a structural mast [5]. This mast normally is supported in the base ground, but it can also be supported in the building structure as well. The mast contains a rack that works as guide or rail. The platform is moved with some motorized pinions. The worker stay at the platform and normally material is lifted with them. Anchoring of the masts is required in every 6 meter [5]. With a single anchorage it can climb up to $12 \mathrm{~m}$ [5].There is normally a gap between the platform and the façade that in case of performing blasting of existing façade elements, this system might not be very adequate because the removed elements fall to the ground.

\subsection{Mobile Cranes}

A crane is mostly used for moving elements from one place to another. There is big variety of cranes, depending on its purpose. The Articulated aerial platform, or the so called "cherry picker", it is a device consisting of a platform that is hold by attached to a hydraulic lifting arm. The arm is mounted onto a vehicle, so it can be moved to the desired spot. This system is mainly used in punctual repair along the building site. There can be many other limitations for a "massive" performance of this crane. One is height limit. Normal cherry pickers reach at most to 12 meters. Another issue is the assembly speed. Probably, for big façade renovation processes, this system is not fast enough, because the material is lifted with the very same crane where the platform is. And finally, the protections systems against material falling are limited to protect the area where the crane is operating.

Another type is the Scissor lift platform. This type of lift is more stable than the precedent one but the operability of the system is always parallel to the ground and façade. The platform can always move due to its electrical motor.

There are also some other methods that are used in a minor way. For instance, Telescoping cranes which operability in building refurbishment is probably reduced.

Besides, the Tower crane can be used also for façade renewal, specially for the positioning of heavy components. Normally another type of scaffolding is required to undertake other purposes[6].

\subsection{Hanging Systems}

Those systems consist of platform that suspends on cables that are fastened from hoists on the top of the building. The hoist can be fixed or can be moved along a rail. The Gondolas [7] are used mainly for maintenance and cleaning purposes. Other lean works such as façade painting can be also undertaken with this system. Gondolas are based on four main parts. The most important element are the cable, the traction hoist, the upper crane placed on the roof of the building and the platform. Nowadays, hoists are powered with electrical motors and they can be controlled easily with manual bottom by the operator in the platform. Cranes can be fixed or can move along a rail installed in the roof. Besides, some of the cranes rotate and along their branches thanks to the hydraulic pumps and electric motors.

Another type is the Suspended scaffolding [7]. This system is a kind of complex gondola crane. It is probably most profitable in high-rises. The structure in the top of the building must be reliable and flat in order to place the hoist and the security devices. The geometry of the building façade shouldn't be very complex.

\subsection{Self-Climbing Devices}

There are some self climbing devices:

Façade cleaning robots. The growing number of buildings with curtain wall façades has lead to find solutions for the cleaning of its external surface. In that sense, the solutions adopted by different companies and research institutes have to be considered [8,9].

Self climbing scaffolding for protection. The only purpose of this device is to protect workers in the top of the building. But it can be a good starting point for developing a new system for building renovation. Even though those devices are used for new building erection, they could be used for renovation processes if some 
adaptation changes are carried out.

Self climbing devices can be independent to the building or interrelated to it, meaning that they can run along rails that are embedded on the façade. They must always work with passive and active security measurements.
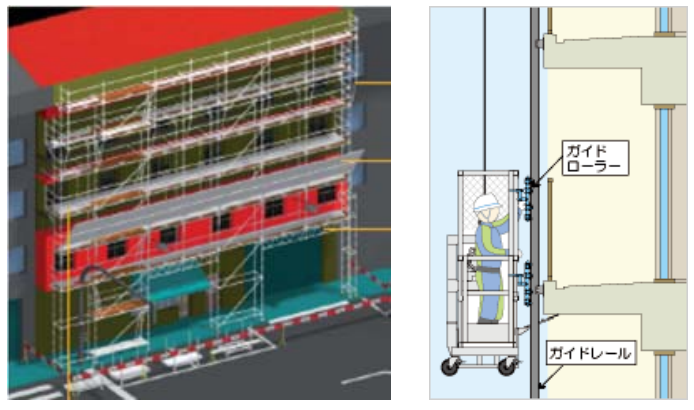

Figure 1. Two examples of existing devices. On the left, fixed scaffolding by ULMA. On the right, Hanging System by BVE Nihon Bisoh.

\subsection{Technology in other fields}

Apart from the many robots that have been used in construction [10], we must focus that there are already similar support systems that are used for automating procedures. One of the closest ones is the so called Automated Storage Systems which consist on moving elements from one side to the other and manipulating it [11].

Besides, it must be taken into account the development of hanging robots [12]. But those similar support systems cannot be used directly for building renovation procedures, they must me adapted and transformed. What we can see on other fields is that a robot is based on interchangeable modular elements and subcomponents.

\section{Development of Automated and Robotic Supporting Systems and Bodies}

Once the existing supporting systems have been briefly analyzed in the previous chapter, it can be stated that the existing technology can be updated towards automated technology. There are some major differences or issues that have to be solved in order to diminish the gap between the existing support systems and Robotic processes.

Basically, the nowadays devices are thought to be operated manually or powered with manual tools. If we have a look on the robotic field, we can separate some functions and parts within a robot: the robot body or mechanical structure, end-effectors, drivers, sensorial devices, actuators, end-effectors and different robotic tools. On the next phase of the research, those appliance will be either customized or expressly designed and fabricated. The robotic body is somehow similar to the existing support system. It provides the physical and mechanical sustenance to the different tasks that are performed. The robot body is the mechanical structure that moves and supports the rest of the devices to certain coordinates.

\subsection{Requirements of the bodies.}

The installation of modern tools and devices into existing support systems doesn't suppose that we get and automated tool. The end-effectors need a suitable body or substructure to work appropriately. The existing support systems must be adapted and modified in order to provide a proper kinematic performance.

There are some requirements that the future automated Supporting System device should accomplish.

Stability: The future device must provide enough Stability for operating with different End-Effectors, Actuators and sensorial Devices. The last advances in some other fields show as that automated devices can work on unstable situations. That is the case of heart surgery, where End-effectors can already work with some movable elements. The sensors can detect unstable situations and the end-effectors adapt constantly to the movements of different organs. But for now this choice is not going to take under consideration because of the cost of those devices.

Security: Any automated device, specially working in a building envelope, must be secure. On the one hand, it must be secure for avoiding any collapse and failure of the system into the buildings' nearby space. On the other hand, the Automated Supporting System has to work without interfering or creating any injure to the workers or to any other people.

Rapid installation: The supporting system must be installed rapidly, besides, the new support system wouldn't be competitive comparing to the traditional systems.

Affordability: Related to this, the overall costs of of the use and performance of the Automated Supporting System must be cheaper than the traditional methods.

Modularity/adaptability towards building typology: The Automated Supporting System must be adaptable to the majority of the buildings, or at least, it should be usable within the same building type. This fact enables a wider coverage of the market and therefore, the system can be more profitable and efficient.

Modularity/adaptability towards building material: The Automated Supporting System should be adaptable to different façade materials. If the future support system is directed towards the installation of just one type of material, let's say, EIFS, the spreading 
of the device might be reduced to some contest. A wide range of the products that it can be installed could enable a better penetration in the market.

As seen before, it seems quite clear that the new support device shouldn't be closely related to the existing devices. That concept might be convenient for the device manufacturing companies because it would suppose less effort for adapting their products. But it might be necessary to go a step further. Not a new design from scratch, but a strong redevelopment. In fact, also redeveloping existing devices towards automation may be short-sighted. Therefore, the future support bodies might follow their own rule and always coordinated with the product type that it is going to be installed. The ROS and Python language will be very useful tools for this purpose.

Another issue that must be undertaken is the direction of the installation process. Should it be Up to down or down to up? If the façade requires blasting and removal, the process should be from up to down. Instead, the newly placed elements would suffer from dust and other small particles. Of course, this happens when both blasting and assembling processes are held at the same time. Besides, for an optimal sealing of the new façade, the horizontal joints have been traditionally more efficiently installed from down to up.

Building renovation is a field were many details have to be taken into account and standard products have to be always adapted. The very same automated process could be also criteria itself for creating or developing new robot bodies or frames. Even more, there could be a mixture of different concepts. The parameterization and adjustment of the component to a specific building façade will has been developed in another phase of the research.

The developed types can be defined as the the scaffolding robot, the robotic crane, the hanging robot, the climbing robot and the bridge crane robot. Within these types, there are infinite possibilities for creating different variants.

Those robot body types are designed for using a similar actuators and end effectors. The definition of this end-effectors and its performing steps ave been carried out in another step of the research.

\subsection{Scaffolding robot}

In the case of the Scaffolding Robot the traditional scaffolding system has been adapted into an automated device, similar to the handling and storage devices. The stability of the supporting systems and the accuracy of the installation of the supporting device are primordial factors. In building renovation, the installation of the scaffolding for instance, may vary some centimetres if the normal procedures are undertaken. For instance, how accurate must the supporting structure be in order to install the automated system? And which is the time and cost needed for installing those devices? The traditional scaffolding system is based on extruded tubes. Those tubes are readapted into rails or guides where the end-effectors can run along.

In the analyzed cases, the scaffolding covers the whole façade, where the robotic working unit is able to move in xyz directions. The placement of the scaffolding may result very laborious (this is an issue that has not been analyzed on the simulation), but once it is installed many robots could operate at the same time and recover a lot of time. As it is similar to a common external scaffolding system, in case of failure of the process, it could be quite easy for a operator to reach the place where the error has occurred.

The scaffolding is based on linear elements that form a 3 dimensional polyedric module. Those elements could be the guides for moving from one place to the other the device with differ rent end effectors.

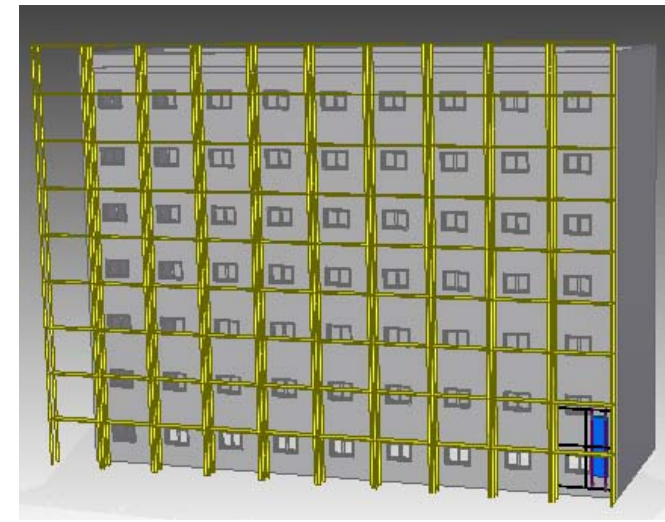

Figure 2. Scaffolding robot performing a simulation.

The uploading of the elements is performed in the inner side; It seems more simple if it is done from the interior size, since it doesn't need to cross the scaffolding itself. The kinematics are simpler this way. The transportation from the inner side requires to separate the scaffolding some centimetres more from the existing façade. Also, an up to down installation process is more convenient.

Within the general frame that offers the supported scaffolding, there can be performed different automation levels. In a semi automated way, between two and four people could be enough for developing the works. One/ two for providing the material in the ground floor, and one/two for placing and anchoring the material to its place.

The tubes of the scaffolding must be tied to the existing wall with cables or tubes. In order to avoid collision with the cables and or tubes, the rails hosted in the tubes of the scaffolding must be duplicated. The 
bridge crane that runs vertically should not touch those cables. The end-effectors or robotic working station should run horizontally too, therefore some connecting bridges must be placed.

\subsection{Crane robot}

Here too, we have two sub-variants. One has been defined as Aerial work robot. The working unit is hosted in the extreme of the arm of the Aerial work platform.

The crane could be installed over a scaffolding in the lower ground, in order to find some protection in dense urban areas. Like in the cherry picker crane, there is a height limitation. But we could say that for buildings of 5-6 floors and 15 meters, there could be no problem. Probably, this crane could be a good pretext for a complete automation of the refurbishment process: Several robotic arms working simultaneously. This robotic crane needs stability and a clear space for its operations. For small façades, a single robotic crane could reach the whole area. This robotic crane is supposed to be mobile. But things change for bigger façades. In these cases, in order to reach the different parts of the façade, the best would be to move the robot along a horizontal rail, parallel to the building, based on the lowest floor. By installing a robotic crane onto scaffolding, the necessary horizontality and stability could be gained. Here also would be the problem of calibrating the device according to the coordinates of the building. Besides, a handicap of this type of element will be the speed of the installation process. The robotic crane would be used for removing the unnecessary material, drilling holes and uploading and fixing the components.

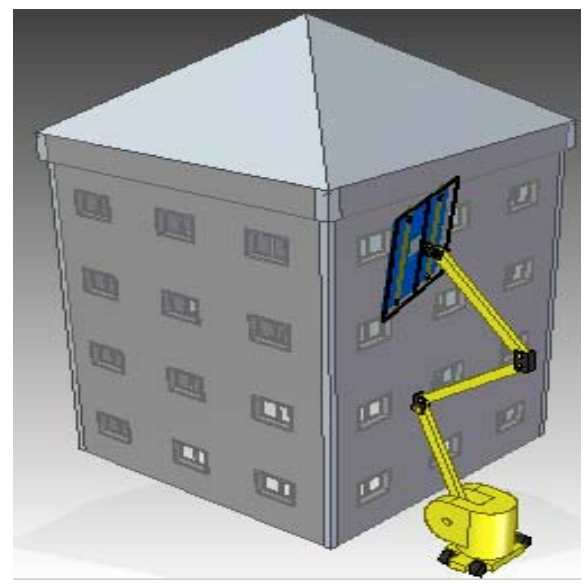

Figure 3. Crane robot performing a simulation.

Nowadays, in order to mechanize big steel elements, we can find big elevator type $\mathrm{CNC}$, similar to a Elevator type robot. Here also there is a limitation on height. The setup of the robot could be very tough and tedious. The problem could be when working with balconies or non planar situations. The weight of this robotic crane could be a big handicap for being installed in the public space because it will need a big substructure as a balance of the reaction forces. It would need the use of cable to triangulate the forces of the elevator.

\subsection{Hanging robot}

The hanging robot is based on the hanging scaffolding. The main issue is that the hanging system suffers the balancing of the platform, unless guides are used.

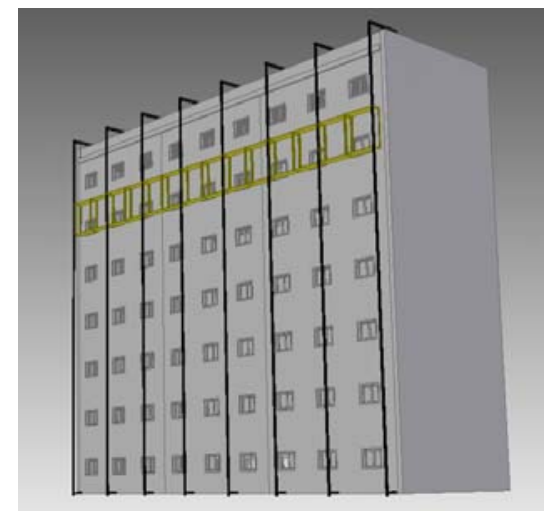

Figure 4. Hanging robot performing a simulation.

Besides, on the subject of installation process, the renovation of building façades using this hanging systems normally goes from up to bottom. It could be interesting if the added component was used as a guide or directive of the platform. Somehow the panel will provide stability and accuracy to the platform. No necessarily rails would be required, but systems such as vacuum lifters could be useful. The platform itself is very appropriate for installing different tools in it. Different end-effectors such as drilling or screw driving units could be adapted. Any other sensor or measuring device could be installed too, if a minimum stability is provided to the platform. Thanks to the configuration of the platform, the person-robot cooperation could be enhanced. In other words, different levels of automation could be possible. This is a very interesting aspect for a gradual insertion of automated devices within the façade upgrading sector. In the end, the suspended systems offer a possibility for being adapted into an automated process.

\subsection{Climbing robot}

The previous works done by the authors where mainly based on this concept [13]. It is a redevelopment of self-climbing formwork systems. The main problem of this system is that loads that are 
transmitted to the façade during the process. If the façade cannot support those loads, the system may fail.

The robot needs to lean on the wall in order to move upstairs and downstairs. Hydraulic devices could be used for that purpose. For safety reasons, the device should be hanged from the top of the building.

The typology of the building interferes in a big degree. The size of the façade component can limit the robotic performance. The roof of the building, if it is not flat roof, might be a problem for some cases.

\subsection{Bridge crane robot based on automated storage systems}

This system could be used either vertically or horizontally. The maximum height of the storage systems spans to 30 meters. First, the special railing and substructure will need to be installed on the top and on the bottom of the building. Those systems are not used outdoors; there must be a protection of the mechatronical devices. The protection system could move along with the vertical bridge and protect it from wind and rain or snow. It could be similar to the protection used for the hanging system, but smaller. The upper and lower guides must be rigid and accurate enough for placing the rails were the bridge crane runs. The normal speed of the crane is $200 \mathrm{~m} / \mathrm{min}$ horizontally and $80 \mathrm{~m} / \mathrm{min}$ vertically. It can upload heavy loads. Normally, the bridge runs horizontally, with one single servomotor in the lower guide. But on the research we have taken into account the possibility of using the bridge crane vertically.

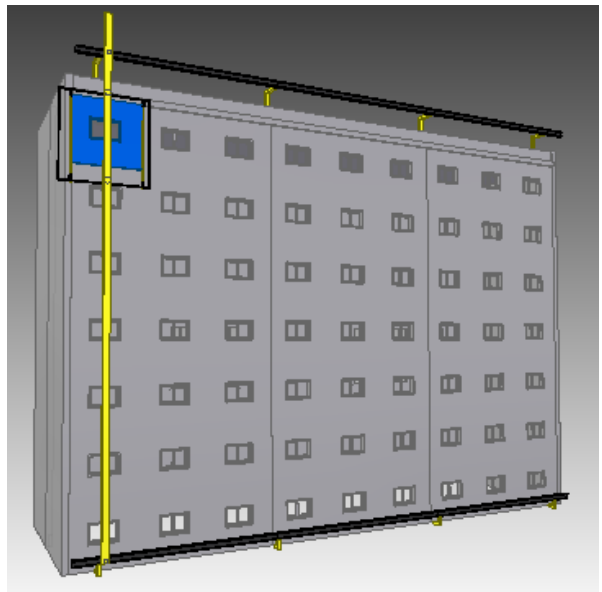

Figure 5. Bridge crane robot performing a simulation.

A similar crane type would be a cable or Suspended cable robotic crane. It will consist on a suspended robot with cables. For installing a suspended robot, probably two guides, one in the top of the building and the other in the lower part should be necessary. Some of the drilling process that is held during the assembly process might be very difficult for this kind of robot, unless the robot itself is tightly held to the façade or other fixed element.

\section{Result analysis}

The robot body itself must be place behind or attached to the building. Depending on the time considered for this step, we have considered the next classification. We can see Scaffolding robot is the type which can take more time.

Table 1 Installation time of different robot bodies

\begin{tabular}{cc}
\hline Type & Installation time \\
\hline Scaffolding robot & high \\
Crane robot & low \\
Hanging robot & moderate \\
Climbing robot & low \\
Bridge crane robot & moderate \\
\hline
\end{tabular}

Depending on the building type, the process can last around one week. On the other hand, the Crane Robot and the Climbing Robot can be ready in a few minutes and hours, depending always on the complexity of then

Once the robot body is placed, the different versions do offer a faster or lower performance during the fixation of the façade component onto the existing building.

Table 2 Performance

\begin{tabular}{cc}
\hline Type & Performance \\
\hline Scaffolding robot & high \\
Crane robot & low \\
Hanging robot & high \\
Climbing robot & low \\
Bridge crane robot & high \\
\hline
\end{tabular}

Finally, the physical limitations of the robot bodies define the use of it in certain building typologies.

Table 3 Building type

\begin{tabular}{cc}
\hline Type & Buielding tupe \\
\hline Scaffolding robot & Mid rise \\
Crane robot & Low/mid rise \\
Hanging robot & High/mid rise \\
Climbing robot & Mid rise \\
Bridge crane robot & Mid rise \\
\hline
\end{tabular}

The crane robot's normally are limited on its height, and therefore are more suitable for mid or low rises.

\section{Conclusion}

A lineal technological development may suggest to gather an improvement of the existing tools and devices. Following a rational scheme, the existing devices could 
install in them end effectors and controls systems in order to achieve a proper façade refurbishment process. There is a concept that could be defined as a progressive automation of the refurbishment process. From completely manual, we should pass to machine assisted, then to semi-automated and finally to fully automated. Nowadays, there is enough basic technology to apply a completely automated construction and renovation process. But it won't be cost effective nowadays.

As said before, some markets might not accept a fully automated robot. The robot could offer different degrees of automations. For that purpose a proper modulation in the robotic is compulsory. The flexibility of the robotic frame should be achieved, both geometrically and on the automation level. There might be also some practical reasons on that. In the end, if the research is directed for gathering a feasible final product, the existing (conservative and traditional) refurbishment industry has to be taken into account, meaning that if any technological proposal is designed from scratch, probably it won't get any interest from the construction lobby. This doesn't mean that ideal scenarios mustn't be taken as an objective. But a down to earth and realistic research may create also interesting output and feedback. Even more, the funding of the research will be probably held with the (financial) support of the existing refurbishment agents.

As a future work, special programming systems such as ROS, Gazebo and Python will be used.

\section{Aknowledgements}

This research has been supported thanks to the AUSMIP+ program. We would like to give special thanks to Prof. Junichiro Maeda, to Prof. Tsuyoshi Seike and to Prof. Soon Wook Kwon for their assistance, dedication and help.

\section{References}

[1] European Commission, A Roadmap for moving to a competitive low carbon economy in 2050, Brussels, 2011

[2] Buildings Performance Institute Europe, Europe's buildings under the microscope. A country-bycountry review of the energy performance of buildings, BPIE, 2011.

[3] Altshuller, G., 40 Principles: TRIZ keys to innovation (Vol. 1). L. Shulyak, \& S. Rodman (Eds.). Technical Innovation Center, Inc., 1997

[4] Ulma Construction. http://www.ulmaconstruction.com/en/projects/rest oration-maintenance-works. Accessed: 02/04/2015.

[5] Electroelsa. http://www.electroelsa.com/en/.
Accessed: 02/04/2015.

[6] BVE Nihon Bisoh. http://www.bisoh.co.jp/. Accessed: 02/04/2015.

[7] Larsen, K. E., et al., Surveying and digital workflow in energy performance retrofit projects using prefabricated elements, Automation in construction, 2011.

[8] Linner, T., Automated and Robotic Construction: Integrated Automated Construction Sites, $\mathrm{PhD}$ Dissertation, TUM, 2013

[9] Bock, T., Construction Robotics, Autonomous Robots Journal, Volume 22, Number 3, pp. 201209, Springer Science + Business Media, USA, 2007

[10] Maeda, J., Current Research and Development and Approach to Future Automated Construction in Japan, Construction Research Congress 2005: pp. $1-11$

[11] Daifuku Co.,On-line: http://www.daifuku.co.jp/, Accessed: 02/04/2015.

[12] Inoue, K., Taisuke, T., Tomohito, T., Arai, T., Omni-directional Gait Of Limb Mechanism Robot Hanging From Grid-like Structure, IEEE/RSJ, 2006

[13] Iturralde. K. Bock T. Robotic Upgrading Of Postwar Social Dwelling Envelops. Proceedings of the ISARC 2013, Montreal, Canada, 2013

[14] Bock, T., Linner, T., Lee, S., Integrated Industrialization Approach for lean Off-/On -site Building Production and Resource Circulation. 7th World Conference on Sustainable Manufacturing, India, 2009 